\title{
Second Order Sufficient Optimality Conditions for a Control Problem with Continuous and Bang-Bang Control Components: Riccati Approach
}

\author{
Nikolai P. Osmolovskii ${ }^{1,2,3, \star}$ and Helmut Maurer ${ }^{4, \star \star}$ \\ 1 Systems Research Institute, Polish Academy of Sciences, \\ ul. Newelska 6, 01-447 Warszawa, Poland \\ 2 Politechnika Radomska, 26-600 Radom, ul. Malczewskiego 20 A, Poland \\ 3 Akademia Podlaska, ul. 3 Maja 54, 08-110 Siedlce, Poland \\ osmolovski@ap.siedlce.pl \\ 4 Westfälische Wilhelms-Universität Münster, \\ Institut für Numerische und Angewandte Mathematik, Einsteinstr. 62, \\ D-48149 Münster, Germany \\ maurer@math.uni-muenster.de
}

\begin{abstract}
Second order sufficient optimality conditions for bang-bang control problems in a very general form have been obtained in [15|21 13]12]1]. These conditions require the positive definiteness (coercivity) of an associated quadratic form on the finite-dimensional critical cone. In the present paper, we investigate similar conditions for optimal control problems with a control variable having two components: a continuous unconstrained control appearing nonlinearly and a bang-bang control appearing linearly and belonging to a convex polyhedron. The coercivity of the quadratic form can be verified by checking solvability of an associated matrix Riccati equation. The results are applied to an economic control problem in optimal production and maintenance, where existing sufficient conditions fail to hold.
\end{abstract}

\section{Introduction}

The classical sufficient second order optimality conditions for an optimization problem with constraints require that the second variation of the Lagrangian be positive definite on the cone of critical directions. In this paper, we investigate sufficient quadratic optimality conditions of such type for optimal control problems with a vector control variable having two components: a continuous unconstrained control appearing nonlinearly in the control system and a bang-bang control appearing linearly and belonging to a convex polyhedron.

In the pure bang-bang case, where all control components appear linearly, second order necessary and sufficient optimality conditions in a very general form have been obtained in Milyutin, Osmolovskii[15], Osmolovskii [21], Agrachev, Stefani, Zezza [1], and Maurer, Osmolovskii [13 12 |24]. Two alternative approaches were developed to establish sufficiency: (1) check the positive definiteness of an associated quadratic form

\footnotetext{
* Supported by the grants RFBR 08-01-00685 and NSh-3233.2008.1.

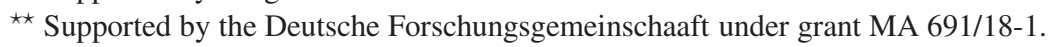

A. Korytowski et al. (Eds.): System Modeling and Optimization, IFIP AICT 312, pp. 411 429, 2009.

(C) IFIP International Federation for Information Processing 2009 
on the finite-dimensional critical cone; (2) verify second-order sufficient conditions for an induced finite-dimensional optimization problem with respect to switching times and free final time. Second order sufficient optimality conditions for control problems where the control variable appears nonlinearly, more precisely, where the strict Legendre condition holds, have been given, e.g., in Malanowski [8], Maurer [9], Maurer, Pickenhain [14], Milyutin, Osmolovskii [15], and Zeidan [28]. Here, one way to check the positive definiteness of the quadratic form is by means of the solvability of an associated Riccati equation.

In this paper, we investigate a class of control problems having two control components, a continuous and a bang-bang component. Such control problems are frequently encountered in practice. Our aim is to obtain second-order sufficient optimality conditions for this class of control problems. It does not come as a surprise that the proof techniques for obtaining sufficient conditions combine the above mentioned methods in the pure bang-bang case and the case where the strict Legendre condition holds. For simplicity, we shall consider control problems on a fixed time interval.

In Sect. 2] we give a statement of the control problem with continuous and bangbang control components (main problem), formulate the minimum principle (first order necessary optimality condition) and introduce the notion of bounded-strong local minimum. In Sect. 3, we present second-order sufficient optimality conditions (SSC) for a bounded-strong minimum in the problem. The main result in Theorem 1 is stated without proof, which is very similar to the proof in the bang-bang case; cf. Milyutin, Osmolovskii [15] and Osmolovskii, Maurer [24], Part 1. Details of the proof will be published elsewhere. In Sect. 4, we give criteria for the positive definiteness of the quadratic form on the critical cone in terms of solutions to a matrix Riccati equation which may be discontinuous at the switching times. In Sect. 5, the main result in Theorem 2 is applied to an economic control problem for optimal production and maintenance which was introduced by Cho, Abad and Parlar [4]. We will show that the numerical solution obtained in Maurer, Kim, and Vossen [10] satisfies the second order test derived in Sect.4 while existing sufficiency results fail to hold.

\section{Control Problem on a Fixed Time Interval}

\subsection{The Main Problem}

Let $x(t) \in \mathbb{R}^{d(x)}$ denote the state variable and $u(t) \in \mathbb{R}^{d(u)}, v(t) \in \mathbb{R}^{d(v)}$ the control variables in the time interval $t \in\left[t_{0}, t_{f}\right]$ with fixed initial time $t_{0}$ and final time $t_{f}$. We shall refer to the following optimal control problem (1)-(4) as the main problem:

$$
\text { Minimize } \quad \mathscr{J}(x(\cdot), u(\cdot), v(\cdot))=J\left(x\left(t_{0}\right), x\left(t_{f}\right)\right)
$$

subject to the constraints

$$
\begin{gathered}
\dot{x}(t)=f(t, x(t), u(t), v(t)), \quad u(t) \in U, \quad(t, x(t), v(t)) \in \mathscr{Q}, \\
F\left(x\left(t_{0}\right), x\left(t_{f}\right)\right) \leq 0, \quad K\left(x\left(t_{0}\right), x\left(t_{f}\right)\right)=0, \quad\left(x\left(t_{0}\right), x\left(t_{f}\right)\right) \in \mathscr{P},
\end{gathered}
$$

where the control variable $u$ appears linearly in the system dynamics,

$$
f(t, x, u, v)=a(t, x, v)+B(t, x, v) u .
$$


Here, $F, K, a$ are column-vector functions, $B$ is a $d(x) \times d(u)$ matrix function, $\mathscr{P} \subset$ $\mathbb{R}^{2 d(x)}, \mathscr{Q} \subset \mathbb{R}^{1+d(x)+d(v)}$ are open sets and $U \subset \mathbb{R}^{d(u)}$ is a convex polyhedron. The functions $J, F, K$ are assumed to be twice continuously differentiable on $\mathscr{P}$ and the functions $a, B$ are twice continuously differentiable on $\mathscr{Q}$. The dimensions of $F, K$ are denoted by $d(F), d(K)$. By $\Delta=\left[t_{0}, t_{f}\right]$ we shall denote the interval of control and use the abbreviations

$$
x_{0}=x\left(t_{0}\right), \quad x_{f}=x\left(t_{f}\right), \quad p=\left(x_{0}, x_{f}\right) .
$$

A process

$$
\Pi=\left\{(x(t), u(t), v(t)) \mid t \in\left[t_{0}, t_{f}\right]\right\}
$$

is said to be admissible, if $x(\cdot)$ is absolutely continuous, $u(\cdot), v(\cdot)$ are measurable and bounded on $\Delta$ and the triple of functions $(x(t), u(t), v(t))$ together with the endpoints $p=\left(x\left(t_{0}\right), x\left(t_{f}\right)\right)$ satisfies the constraints (2) and (3).

Definition 1. An admissible process $\Pi$ affords a Pontryagin local minimum, if for each compact set $\mathscr{C} \subset \mathscr{Q}$ there exists $\varepsilon>0$ such that $\mathscr{J}(\tilde{\Pi}) \geq \mathscr{J}(\Pi)$ for all admissible processes $\tilde{\Pi}=\left\{(\tilde{x}(t), \tilde{u}(t), \tilde{v}(t)) \mid t \in\left[t_{0}, t_{1}\right]\right\}$ such that:

(a) $\max _{\Delta}|\tilde{x}(t)-x(t)|<\varepsilon$;

(b) $\int_{\Delta}^{\Delta}|\tilde{u}(t)-u(t)| d t<\varepsilon ; \quad \int_{\Delta}|\tilde{v}(t)-v(t)| d t<\varepsilon$;

(c) $(t, \tilde{x}(t), \tilde{v}(t)) \in \mathscr{C}$ a.e. on $\Delta$.

\subsection{First Order Necessary Optimality Conditions}

Let

$$
\Pi=\left\{(x(t), u(t), v(t)) \mid t \in\left[t_{0}, t_{f}\right]\right\}
$$

be a fixed admissible process such that the control $u(t)$ is a piecewise constant function and the control $v(t)$ is a continuous function on the interval $\Delta=\left[t_{0}, t_{f}\right]$. In order to make the notations simpler, we do not use such symbols and indices as zero, hat or asterisk to distinguish this trajectory from others. Denote by

$$
\theta=\left\{t_{1}, \ldots, t_{s}\right\}, \quad t_{0}<t_{1}<\cdots<t_{s}<t_{f},
$$

the finite set of all discontinuity points (jump points) of the control $u(t)$. Then $\dot{x}(t)$ is a piecewise continuous function whose discontinuity points belong to $\theta$, and hence $x(t)$ is a piecewise smooth function on $\Delta$. Henceforth, we shall use the notation

$$
[u]^{k}=u^{k+}-u^{k-}
$$

to denote the jump of the function $u(t)$ at the point $t_{k} \in \theta$, where

$$
u^{k-}=u\left(t_{k}-0\right), \quad u^{k+}=u\left(t_{k}+0\right)
$$

are the left hand and the right hand values of the control $u(t)$ at $t_{k}$, respectively. Similarly, we denote by $[\dot{x}]^{k}$ the jump of the function $\dot{x}(t)$ at the point $t_{k}$. 
Let us formulate first-order necessary conditions of a Pontryagin minimum for the process $\Pi$ in the form of the Pontryagin minimum principle. To this end we introduce the Pontryagin or Hamiltonian function

$$
H(t, x, \psi, u, v)=\psi f(t, x, u, v)=\psi a(t, x, v)+\psi B(t, x, v) u,
$$

where $\psi$ is a row vector of dimension $d(\psi)=d(x)$, while $x, u, f, F$ and $K$ are column vectors. The row vector of dimension $d(u)$,

$$
\sigma(t, x, \psi, v)=\psi B(t, x, v)
$$

will be called the switching function for the u-component of the control. Denote by $l$ the endpoint Lagrange function

$$
l\left(\alpha_{0}, \alpha, \beta, p\right)=\alpha_{0} J(p)+\alpha F(p)+\beta K(p), \quad p=\left(x_{0}, x_{f}\right),
$$

where $\alpha$ and $\beta$ are row-vectors with $d(\alpha)=d(F), d(\beta)=d(K)$, and $\alpha_{0}$ is a number. We introduce a tuple of Lagrange multipliers

$$
\lambda=\left(\alpha_{0}, \alpha, \beta, \psi(\cdot)\right)
$$

such that

$$
\psi(\cdot): \Delta \rightarrow \mathbb{R}^{d(x)}
$$

is continuous on $\Delta$ and continuously differentiable on each interval of the set $\Delta \backslash \theta$. In the sequel, we shall denote first or second order partial derivatives by subscripts referring to the variables.

Denote by $M_{0}$ the set of the normalized tuples $\lambda$ satisfying the minimum principle conditions for the process $\Pi$ :

$$
\begin{gathered}
\alpha_{0} \geq 0, \alpha \geq 0, \alpha F(p)=0, \alpha_{0}+\sum \alpha_{i}+\sum\left|\beta_{j}\right|=1, \\
\dot{\psi}=-H_{x}, \quad \forall t \in \Delta \backslash \theta, \\
\psi\left(t_{0}\right)=-l_{x_{0}}, \quad \psi\left(t_{f}\right)=l_{x_{f}}, \\
H(t, x(t), \psi(t), u, v) \geq H(t, x(t), \psi(t), u(t), v(t)) \\
\text { for all } t \in \Delta \backslash \theta, u \in U, v \in \mathbb{R}^{d(v)} \text { such that }(t, x(t), v) \in \mathscr{Q} \text {. }
\end{gathered}
$$

The derivatives $l_{x_{0}}$ and $l_{x_{f}}$ are taken at the point $\left(\alpha_{0}, \alpha, \beta, p\right)$, where $p=\left(x\left(t_{0}\right), x\left(t_{f}\right)\right)$, and the derivative $H_{x}$ is evaluated at the point

$$
(t, x(t), \psi(t), u(t), v(t)), \quad t \in \Delta \backslash \theta .
$$

The condition $M_{0} \neq \emptyset$ constitutes a first-order necessary condition of a Pontryagin minimum for the process $\Pi$ which is called the Pontryagin minimum principle, cf., Pontryagin et al. [25], Hestenes [7], Milyutin, Osmolovskii [15]. The set $M_{0}$ is a finitedimensional compact set and the projector $\lambda \mapsto\left(\alpha_{0}, \alpha, \beta\right)$ is injective on $M_{0}$.

In the sequel, it will be convenient to use the abbreviation $(t)$ for indicating all arguments $(t, x(t), \psi(t), u(t), v(t))$, e.g.,

$$
H(t)=H(t, x(t), \psi(t), u(t), v(t)), \quad \sigma(t)=\sigma(t, x(t), \psi(t), v(t)) .
$$


Let $\lambda=\left(\alpha_{0}, \alpha, \beta, \psi(\cdot)\right) \in M_{0}$. It is well-known that $H(t)$ is a continuous function. In particular, $[H]^{k}=H^{k+}-H^{k-}=0$ holds for each $t_{k} \in \theta$, where $H^{k-}:=H\left(t_{k}-0\right)$ and $H^{k+}:=H\left(t_{k}+0\right)$. We shall denote by $H^{k}$ the common value of $H^{k-}$ and $H^{k+}$ :

$$
H^{k}:=H^{k-}=H^{k+} .
$$

The relations

$$
[H]^{k}=0, \quad[\psi]^{k}=0, \quad k=1, \ldots, s,
$$

constitute the Weierstrass-Erdmann conditions for a broken extremal. We formulate one more condition of this type which will be important for the statement of second order conditions for extremal with jumps in the control. Namely, for $\lambda \in M_{0}$ and $t_{k} \in \theta$ consider the function

$$
\begin{aligned}
\left(\Delta_{k} H\right)(t) & =H\left(t, x(t), \psi(t), u^{k+}, v\left(t_{k}\right)\right)-H\left(t, x(t), \psi(t), u^{k-}, v\left(t_{k}\right)\right) \\
& =\sigma\left(t, x(t), \psi(t), v\left(t_{k}\right)\right)[u]^{k} .
\end{aligned}
$$

Proposition 1. For each $\lambda \in M_{0}$ the following equalities hold

$$
\left.\frac{d}{d t}\left(\Delta_{k} H\right)\right|_{t=t_{k}-0}=\left.\frac{d}{d t}\left(\Delta_{k} H\right)\right|_{t=t_{k}+0}, \quad k=1, \ldots, s .
$$

Consequently, for each $\lambda \in M_{0}$ the function $\left(\Delta_{k} H\right)(t)$ has a derivative at the point $t_{k} \in \theta$. In the sequel, we will consider the quantities

$$
D^{k}(H)=-\frac{d}{d t}\left(\Delta_{k} H\right)\left(t_{k}\right), \quad k=1, \ldots, s .
$$

Then the minimum condition (19) implies the following property:

Proposition 2. For each $\lambda \in M_{0}$ the following conditions hold:

$$
D^{k}(H) \geq 0, \quad k=1, \ldots, s .
$$

Note that the value $D^{k}(H)$ also can be written in the form

$$
D^{k}(H)=-H_{x}^{k+} H_{\psi}^{k-}+H_{x}^{k-} H_{\psi}^{k+}-\left[H_{t}\right]^{k}=\dot{\psi}^{k+} \dot{x}^{k-}-\dot{\psi}^{k-} \dot{x}^{k+}+\left[\psi_{0}\right]^{k}
$$

where $H_{x}^{k-}$ and $H_{x}^{k+}$ are the left- and the right-hand values of the function $H_{x}(t)$ at $t_{k}$, respectively, $\left[H_{t}\right]^{k}$ is the jump of the function $H_{t}(t)$ at $t_{k}$, etc., and $\psi_{0}(t)=-H(t)$.

\subsection{Integral Cost Function, Unessential Variables, Bounded-Strong Minimum}

It is well-known that any control problem with a cost functional in integral form

$$
\mathscr{J}=\int_{t_{0}}^{t_{f}} f_{0}(t, x(t), u(t), v(t)) d t
$$


can be reduced to the form (1) by introducing a new state variable $y$ defined by the state equation

$$
\dot{y}=f_{0}(t, x, u, v), \quad y\left(t_{0}\right)=0 .
$$

This yields the cost function $\mathscr{J}=y\left(t_{f}\right)$. The control variable $u$ is assumed to appear linearly in the function $f_{0}$,

$$
f_{0}(t, x, u, v)=a_{0}(t, x, v)+B_{0}(t, x, v) u .
$$

The component $y$ is called an unessential component in the augmented problem. The general definition of an unessential component is as follows.

Definition 2. The $i$-th component $x_{i}$ of the state vector $x$ is called unessential if the function $f$ does not depend on $x_{i}$ and if the functions $F, J, K$ are affine in $x_{i 0}=x_{i}\left(t_{0}\right)$ and $x_{i f}=x_{i}\left(t_{f}\right)$.

In the following, let $\underline{x}$ denote the vector of all essential components of state vector $x$.

Definition 3. The process $\Pi$ affords a bounded-strong minimum, if for each compact set $\mathscr{C} \subset \mathscr{Q}$ there exists $\varepsilon>0$ such that $\mathscr{J}(\widetilde{\Pi}) \geq \mathscr{J}(\Pi)$ for all admissible processes $\widetilde{\Pi}=\left\{(\tilde{x}(t), \tilde{u}(t), \tilde{v}(t)) \mid t \in\left[t_{0}, t_{f}\right]\right\}$ such that

(a) $\left|\tilde{x}\left(t_{0}\right)-x\left(t_{0}\right)\right|<\varepsilon$,

(b) $\max _{\Delta}|\underline{\tilde{x}}(t)-\underline{x}(t)|<\varepsilon$,

(c) $(t, \tilde{x}(t), \tilde{v}(t)) \in \mathscr{C}$ a.e. on $\Delta$.

The strict bounded-strong minimum is defined in a similar way, with the non-strict inequality $\mathscr{J}(\widetilde{\Pi}) \geq \mathscr{J}(\Pi)$ replaced by the strict one and the process $\widetilde{\Pi}$ required to be different from $\Pi$.

\section{Quadratic Sufficient Optimality Conditions}

In this section, we shall formulate a quadratic sufficient optimality condition for a bounded-strong minimum (Definition 3) for given control process. This quadratic condition is based on the properties of a quadratic form on the so-called critical cone, whose elements are first order variations along a given process $\Pi$.

\subsection{Critical Cone}

For a given process $\Pi$ we introduce the space $\mathscr{Z}^{2}(\theta)$ and the critical cone $\mathscr{K} \subset$ $\mathscr{Z}^{2}(\theta)$. Denote by $P_{\theta} W^{1,2}\left(\Delta, \mathbb{R}^{d(x)}\right)$ the space of piecewise continuous functions $\bar{x}(\cdot)$ : $\Delta \rightarrow \mathbb{R}^{d(x)}$, which are absolutely continuous on each interval of the set $\Delta \backslash \theta$ and have a square integrable first derivative. By $L^{2}\left(\Delta, \mathbb{R}^{d(v)}\right)$ we denote the space of square integrable functions $\bar{v}(\cdot): \Delta \rightarrow \mathbb{R}^{d(v)}$. For each $\bar{x} \in P_{\theta} W^{1,2}\left(\Delta, \mathbb{R}^{d(x)}\right)$ and for $t_{k} \in \theta$ we set

$$
\bar{x}^{k-}=\bar{x}\left(t_{k}-0\right), \quad \bar{x}^{k+}=\bar{x}\left(t_{k}+0\right), \quad[\bar{x}]^{k}=\bar{x}^{k+}-\bar{x}^{k-} .
$$


Let $\bar{z}=(\xi, \bar{x}, \bar{v})$, where $\xi \in \mathbb{R}^{s}, \bar{x} \in P_{\theta} W^{1,2}\left(\Delta, \mathbb{R}^{d(x)}\right), \bar{v} \in L^{2}\left(\Delta, \mathbb{R}^{d(v)}\right)$. Thus,

$$
\bar{z} \in \mathscr{Z}^{2}(\theta):=\mathbb{R}^{s} \times P_{\theta} W^{1,2}\left(\Delta, \mathbb{R}^{d(x)}\right) \times L^{2}\left(\Delta, \mathbb{R}^{d(v)}\right) .
$$

For each $\bar{z}$ we set

$$
\bar{x}_{0}=\bar{x}\left(t_{0}\right), \quad \bar{x}_{f}=\bar{x}\left(t_{f}\right), \quad \bar{p}=\left(\bar{x}_{0}, \bar{x}_{f}\right) .
$$

The vector $\bar{p}$ is considered as a column vector. Denote by

$$
I_{F}(p)=\left\{i \in\{1, \ldots, d(F)\} \mid F_{i}(p)=0\right\}
$$

the set of indices of all active endpoint inequalities $F_{i}(p) \leq 0$ at the point $p=\left(x\left(t_{0}\right)\right.$, $\left.x\left(t_{f}\right)\right)$. Denote by $\mathscr{K}$ the set of all $\bar{z} \in \mathscr{Z}^{2}(\theta)$ satisfying the following conditions:

$$
\begin{gathered}
J^{\prime}(p) \bar{p} \leq 0, \quad F_{i}^{\prime}(p) \bar{p} \leq 0 \forall i \in I_{F}(p), \quad K^{\prime}(p) \bar{p}=0, \\
\dot{\bar{x}}(t)=f_{x}(t) \bar{x}(t)+f_{v}(t) \bar{v}(t), \\
{[\bar{x}]^{k}=[\dot{x}]^{k} \xi_{k}, \quad k=1, \ldots, s,}
\end{gathered}
$$

where $p=\left(x\left(t_{0}\right), x\left(t_{f}\right)\right)$ and $[\dot{x}]^{k}=\dot{x}\left(t_{k}+0\right)-\dot{x}\left(t_{k}-0\right)$. It is obvious that $\mathscr{K}$ is a convex cone in the Hilbert space $\mathscr{Z}^{2}(\theta)$ with finitely many faces. We call $\mathscr{K}$ the critical cone.

\subsection{Quadratic Form}

Let us introduce a quadratic form on the critical cone $\mathscr{K}$ defined by the conditions (36)-(38). For each $\lambda \in M_{0}$ and $\bar{z} \in \mathscr{K}$ we set

$$
\begin{aligned}
\Omega(\lambda, \bar{z}) & =\left\langle l_{p p}(p) \bar{p}, \bar{p}\right\rangle+\sum_{k=1}^{s}\left(D^{k}(H) \xi_{k}^{2}-[\dot{\psi}]^{k} \bar{x}_{\mathrm{av}}^{k} \bar{\xi}_{k}\right) \\
& +\int_{t_{0}}^{t_{f}}\left(\left\langle H_{x x}(t) \bar{x}(t), \bar{x}(t)\right\rangle+2\left\langle H_{x v}(t) \bar{v}(t), \bar{x}(t)\right\rangle+\left\langle H_{v v}(t) \bar{v}(t), \bar{v}(t)\right\rangle\right) d t,
\end{aligned}
$$

where

$$
\begin{gathered}
l_{p p}(p)=l_{p p}\left(\alpha_{0}, \alpha, \beta, p\right), p=\left(x\left(t_{0}\right), x\left(t_{f}\right)\right), \bar{x}_{\mathrm{av}}^{k}=\frac{1}{2}\left(\bar{x}^{k-}+\bar{x}^{k+}\right), \\
H_{x x}(t)=H_{x x}(t, x(t), \psi(t), u(t), v(t)), \quad \text { etc. }
\end{gathered}
$$

Note that the functional $\Omega(\lambda, \bar{z})$ is linear in $\lambda$ and quadratic in $\bar{z}$.

\subsection{Quadratic Sufficient Optimality Conditions}

Denote by $M_{0}^{+}$the set of all $\lambda \in M_{0}$ satisfying the conditions:

(a) $H(t, x(t), \psi(t), u, v)>H(t, x(t), \psi(t), u(t), v(t))$ for all $t \in \Delta \backslash \theta, u \in U$, $v \in \mathbb{R}^{d(v)}$, such that $(t, x(t), v) \in \mathscr{Q}$ and $(u, v) \neq(u(t), v(t))$;

(b) $H\left(t_{k}, x\left(t_{k}\right), \psi\left(t_{k}\right), u, v\right)>H^{k} \quad$ for all $t_{k} \in \theta, \quad u \in U, v \in \mathbb{R}^{d(v)} \quad$ such that $\left(t_{k}, x\left(t_{k}\right), v\right) \in \mathscr{Q},(u, v) \neq\left(u\left(t_{k}-0\right), v\left(t_{k}\right)\right),(u, v) \neq\left(u\left(t_{k}+0\right), v\left(t_{k}\right)\right)$, where $H^{k}:=$ $H^{k-}=H^{k+}$. 
Let Arg $\min _{\tilde{u} \in U} \sigma(t) \tilde{u}$ be the set of points $v \in U$ where the minimum of the linear function $\sigma(t) \tilde{u}$ is attained.

Definition 4. For a given admissible process $\Pi$ with a piecewise constant control $u(t)$ and continuous control $v(t)$ we say that $u(t)$ is a strict bang-bang control, if the set $M_{0}$ is nonempty and there exists $\lambda \in M_{0}$ such that

$$
\operatorname{Arg} \min _{\tilde{u} \in U} \sigma(t) \tilde{u}=[u(t-0), u(t+0)]
$$

where $[u(t-0), u(t+0)]$ denotes the line segment spanned by the vectors $u(t-0)$ and $u(t+0)$.

If $\operatorname{dim}(u)=1$, then the strict bang-bang property is equivalent to $\sigma(t) \neq 0$ for all $t \in$ $\Delta \backslash \theta$. For $\operatorname{dim}(u)>1$ the strict bang-bang property requires that two or more control components do not switch simultaneously and the components of the switching vector function vanish only at the switching points. If the set $M_{0}^{+}$is nonempty, then, obviously, $u(t)$ is a strict bang-bang control.

Definition 5. An element $\lambda \in M_{0}$ is said to be strictly Legendrian if the following conditions are satisfied:

(a) for each $t \in \Delta \backslash \theta$ the quadratic form $\left\langle H_{v v}(t, x(t), \psi(t), u(t), v(t)) \bar{v}, \bar{v}\right\rangle$ is positive definite on $\mathbb{R}^{d(v)}$;

(b) for each $t_{k} \in \theta$ the quadratic form $\left\langle H_{v v}\left(t_{k}, x\left(t_{k}\right), \psi\left(t_{k}\right), u\left(t_{k}-0\right), v\left(t_{k}\right)\right) \bar{v}, \bar{v}\right\rangle$ is positive definite on $\mathbb{R}^{d(v)}$;

(c) for each $t_{k} \in \theta$ the quadratic form $\left\langle H_{v v}\left(t_{k}, x\left(t_{k}\right), \psi\left(t_{k}\right), u\left(t_{k}+0\right), v\left(t_{k}\right)\right) \bar{v}, \bar{v}\right\rangle$ is positive definite on $\mathbb{R}^{d(v)}$;

(d) $D^{k}(H)>0$ for all $t_{k} \in \theta$.

Let $\operatorname{Leg}_{+}\left(M_{0}^{+}\right)$be the set of all strictly Legendrian elements $\lambda \in M_{0}^{+}$and put

$$
\bar{\gamma}(\bar{z})=\langle\xi, \xi\rangle+\left\langle\bar{x}\left(t_{0}\right), \bar{x}\left(t_{0}\right)\right\rangle+\int_{t_{0}}^{t_{f}}\langle\bar{v}(t), \bar{v}(t)\rangle d t .
$$

Theorem 1. Let the following Condition $\mathscr{B}$ be fulfilled for the process $\Pi$ :

(a) the set $\operatorname{Leg}_{+}\left(M_{0}^{+}\right)$is nonempty, hence, in particular $u(t)$ is a strict bang-bang control;

(b) there exists a nonempty compact set $M \subset \operatorname{Leg}_{+}\left(M_{0}^{+}\right)$and a number $C>0$ such that $\max _{\lambda \in M} \Omega(\lambda, \bar{z}) \geq C \bar{\gamma}(\bar{z})$ for all $\bar{z} \in \mathscr{K}$.

Then $\Pi$ is a strict bounded-strong minimum.

Remark. If the set $\operatorname{Leg}_{+}\left(M_{0}^{+}\right)$is nonempty and $\mathscr{K}=\{0\}$, then (b) is fulfilled automatically. This case can be considered as a first order sufficient optimality condition for a strict bounded-strong minimum.

The proof of Theorem 1 is very similar to the proof of the sufficient quadratic optimality condition for the pure bang-bang case given in Milyutin, Osmolovskii [15] Theorem 12.2, p. 302 and Osmolovskii, Maurer [24], Part 1. The proof is based on the SSC for broken extremals in the general problem of calculus of variations; see Osmolovskii [21]. 


\section{Riccati Approach}

The following question suggests itself from a numerical point of view: how does the numerical check of the quadratic sufficient optimality conditions in Theorem 1 proceed? For simplicity, we shall assume that (a) the initial value $x\left(t_{0}\right)$ is fixed and (b) there are no endpoint constraints of inequality type. Assumptions (a) and (b) will simplify the boundary conditions for the solution of the associated Riccati equation. Thus we consider a problem:

$$
\text { Minimize } J\left(x\left(t_{f}\right)\right)
$$

under the constraints

$$
x\left(t_{0}\right)=x_{0}, \quad K\left(x\left(t_{f}\right)\right)=0, \quad \dot{x}=f(t, x, u, v), \quad u \in U,
$$

where

$$
f(t, x, u, v)=a(t, x, v)+B(t, x, v) u,
$$

$U \subset \mathbb{R}^{d(u)}$ is a convex polyhedron and $J, K, B$ are $C^{2}$-functions. In the sequel, we shall assume that there exists $\lambda \in M_{0}$ such that $\alpha_{0}>0$.

\subsection{Critical Cone $\mathscr{K}$ and Quadratic Form $\Omega$}

For this problem, the critical cone is a subspace which is defined by the relations

$$
\begin{gathered}
\bar{x}\left(t_{0}\right)=0, \quad K_{x_{f}}(p) \bar{x}\left(t_{f}\right)=0, \\
\dot{\bar{x}}(t)=f_{x}(t) \bar{x}(t)+f_{v}(t) \bar{v}(t), \quad[\bar{x}]^{k}=[\dot{x}]^{k} \xi_{k}, \quad k=1, \ldots, s .
\end{gathered}
$$

These relations imply that $J^{\prime}(p) \bar{p}=0$ since $\alpha_{0}>0$. Hence, the quadratic form is given by

$$
\begin{aligned}
\Omega(\lambda, \bar{z}) & =\left\langle l_{x_{f} x_{f}}(p) \bar{x}_{f}, \bar{x}_{f}\right\rangle+\sum_{k=1}^{s}\left(D^{k}(H) \xi_{k}^{2}-2[\dot{\psi}]^{k} \bar{x}_{\mathrm{av}}^{k} \xi_{k}\right) \\
& +\int_{t_{0}}^{t_{f}}\left(\left\langle H_{x x}(t) \bar{x}(t), \bar{x}(t)\right\rangle+2\left\langle H_{x v}(t) \bar{v}(t), \bar{x}(t)\right\rangle+\left\langle H_{v v}(t) \bar{v}(t), \bar{v}(t)\right\rangle\right) d t,
\end{aligned}
$$

where, by definition, $\bar{x}_{f}=\bar{x}\left(t_{f}\right)$. We assume that there exists $\lambda \in M_{0}^{+}$such that

$$
D^{k}(H)>0, \quad k=1, \ldots, s,
$$

and the strengthened Legendre condition is satisfied with respect to $v$ :

$$
\left\langle H_{v v}(t) \bar{v}, \bar{v}\right\rangle \geq c\langle\bar{v}, \bar{v}\rangle \quad \forall \bar{v} \in \mathbb{R}^{d(v)}, \quad \forall t \in\left[t_{0}, t_{f}\right] \backslash \theta \quad(c>0) .
$$

From now on we shall fix $\lambda \in M_{0}^{+}$with these properties. 


\section{2 $Q$-Transformation of $\Omega$ on $\mathscr{K}$}

Set $n=d(x)$ and let $Q(t)$ be a symmetric $n \times n$ matrix on $\left[t_{0}, t_{f}\right]$ with piecewise continuous entries that are absolutely continuous on each interval of the set $\left[t_{0}, t_{f}\right] \backslash \theta$. For each $\bar{z} \in \mathscr{K}$ we obviously have

$$
\int_{t_{0}}^{t_{f}} \frac{d}{d t}\langle Q \bar{x}, \bar{x}\rangle d t=\left.\langle Q \bar{x}, \bar{x}\rangle\right|_{t_{0}} ^{t_{f}}-\sum_{k=1}^{s}[\langle Q \bar{x}, \bar{x}\rangle]^{k},
$$

where $[\langle Q \bar{x}, \bar{x}\rangle]^{k}$ is the jump of the function $\langle Q \bar{x}, \bar{x}\rangle$ at the point $t_{k} \in \theta$. Using the equation $\dot{\bar{x}}=f_{x} \bar{x}+f_{v} \bar{v}$ and the initial condition $\bar{x}\left(t_{0}\right)=0$, we obtain

$$
\begin{aligned}
& -\left\langle Q\left(t_{f}\right) \bar{x}_{f}, \bar{x}_{f}\right\rangle+\sum_{k=1}^{s}[\langle Q \bar{x}, \bar{x}\rangle]^{k} \\
& +\int_{t_{0}}^{t_{f}}\left(\langle\dot{Q} \bar{x}, \bar{x}\rangle+\left\langle Q\left(f_{x} \bar{x}+f_{v} \bar{v}\right), \bar{x}\right\rangle+\left\langle Q \bar{x}, f_{x} \bar{x}+f_{v} \bar{v}\right\rangle\right) d t=0 .
\end{aligned}
$$

Adding this zero term to the form $\Omega(\lambda, \bar{z})$ in $(49)$ we get

$$
\begin{aligned}
\Omega(\lambda, \bar{z}) & =\left\langle\left(l_{x_{f} x_{f}}-Q\left(t_{f}\right)\right) \bar{x}_{f}, \bar{x}_{f}\right\rangle+\sum_{k=1}^{s}\left(D^{k}(H) \xi_{k}^{2}-2[\dot{\psi}]^{k} \bar{x}_{\mathrm{av}}^{k} \xi_{k}+[\langle Q \bar{x}, \bar{x}\rangle]^{k}\right) \\
& +\int_{t_{0}}^{t_{f}}\left(\left\langle\left(H_{x x}+\dot{Q}+Q f_{x}+f_{x}^{T} Q\right) \bar{x}, \bar{x}\right\rangle+\left\langle\left(H_{x v}+Q f_{v}\right) \bar{v}, \bar{x}\right\rangle\right. \\
& \left.+\left\langle\left(H_{v x}+f_{v}^{T} Q\right) \bar{x}, \bar{v}\right\rangle+\left\langle H_{v v}(t) \bar{v}(t), \bar{v}(t)\right\rangle\right) d t .
\end{aligned}
$$

We call this formula the $Q$-transformation of $\Omega$ on $\mathscr{K}$.

\subsection{Transformation of $\Omega$ on $\mathscr{K}$ to Perfect Squares}

In order to transform the integral term in $\Omega(\lambda, \bar{z})$ to a perfect square we assume that $Q(t)$ satisfies the following matrix Riccati equation; cf. also [9]14|28]:

$$
\dot{Q}+Q f_{x}+f_{x}^{T} Q+H_{x x}-\left(H_{x v}+Q f_{v}\right) H_{v v}^{-1}\left(H_{v x}+f_{v}^{T} Q\right)=0 .
$$

Then the integral term in $\Omega$ can be written as

$$
\int_{t_{0}}^{t_{f}}\left\langle H_{v v}^{-1} \bar{h}, \bar{h}\right\rangle d t, \text { where } \bar{h}=\left(H_{v x}+f_{v}^{T} Q\right) \bar{x}+H_{v v} \bar{v} .
$$

A remarkable fact is that the terms

$$
\omega_{k}:=D^{k}(H) \xi_{k}^{2}-2[\dot{\psi}]^{k} \bar{x}_{\mathrm{av}}^{k} \xi_{k}+[\langle Q \bar{x}, \bar{x}\rangle]^{k}
$$


can also be transformed to perfect squares if the matrix $Q(t)$ satisfies a special jump condition at each point $t_{k} \in \theta$. This jump condition was obtained in Osmolovskii, Lempio [22]. Namely, for each $k=1, \ldots, s$ put

$$
\begin{gathered}
Q^{k-}=Q\left(t_{k}-0\right), \quad Q^{k+}=Q\left(t_{k}+0\right), \quad[Q]^{k}=Q^{k+}-Q^{k-}, \\
q_{k-}=\left([\dot{x}]^{k}\right)^{T} Q^{k-}-[\dot{\psi}]^{k}, \quad b_{k-}=D^{k}(H)-\left(q_{k-}\right)[\dot{x}]^{k},
\end{gathered}
$$

where $[\dot{x}]^{k}$ is a column vector, while $q_{k-},\left([\dot{x}]^{k}\right)^{T}$ and $[\dot{\psi}]^{k}$ are row vectors, and $b_{k-}$ is a number. We shall assume that

$$
b_{k-}>0, k=1, \ldots, s,
$$

holds and that $Q$ satisfies the following jump conditions

$$
[Q]^{k}=\left(b_{k-}\right)^{-1}\left(q_{k-}\right)^{T}\left(q_{k-}\right),
$$

where $\left(q_{k-}\right)$ is a row vector, $\left(q_{k-}\right)^{T}$ is a column vector and hence $\left(q_{k-}\right)^{T}\left(q_{k-}\right)$ is a symmetric $n \times n$ matrix. Then one can show (see [22]) that

$$
\omega_{k}=\left(b_{k-}\right)^{-1}\left(\left(b_{k-}\right) \xi_{k}+\left(q_{k-}\right)\left(\bar{x}^{k+}\right)\right)^{2}=\left(b_{k-}\right)^{-1}\left(D^{k}(H) \xi_{k}+\left(q_{k-}\right)\left(\bar{x}^{k-}\right)\right)^{2} .
$$

Therefore, we obtain the following transformation of the quadratic form $\Omega=\Omega(\lambda, \bar{z})$ to perfect squares on the critical cone $\mathscr{K}$ :

$$
\begin{aligned}
\Omega & =\left\langle\left(l_{x_{f} x_{f}}-Q\left(t_{f}\right)\right) \bar{x}_{f}, \bar{x}_{f}\right\rangle+\sum_{k=1}^{s}\left(b_{k-}\right)^{-1}\left(D^{k}(H) \xi_{k}+\left(q_{k-}\right)\left(\bar{x}^{k-}\right)\right)^{2} \\
& +\int_{t_{0}}^{t_{f}}\left\langle H_{v v}^{-1} \bar{h}, \bar{h}\right\rangle d t
\end{aligned}
$$

where

$$
\bar{h}=\left(H_{v x}+f_{v}^{T} Q\right) \bar{x}+H_{v v} \bar{v} .
$$

In addition, let us assume that

$$
\left\langle\left(l_{x_{f} x_{f}}-Q\left(t_{f}\right)\right) \bar{x}_{f}, \bar{x}_{f}\right\rangle \geq 0
$$

for all $\bar{x}_{f} \in \mathbb{R}^{d(x)}$ such that

$$
K_{x_{f}}\left(x\left(t_{f}\right)\right) \bar{x}_{f}=0 .
$$

Then, obviously, $\Omega(\lambda, \bar{z}) \geq 0$ on $\mathscr{K}$. Let us show now that $\Omega(\lambda, \bar{z})>0$ for each nonzero element $\bar{z} \in \mathscr{K}$. This means that $\Omega(\lambda, \bar{z})$ is positive definite on the critical cone $\mathscr{K}$ since $\Omega(\lambda, \bar{z})$ is a Legendrian quadratic form.

Assume that $\Omega(\lambda, \bar{z})=0$ for some element $\bar{z} \in \mathscr{K}$. Then, for this element, the following equations hold

$$
\begin{aligned}
& \bar{x}\left(t_{0}\right)=0, \\
& D^{k}(H) \xi_{k}+\left(q_{k-}\right)\left(\bar{x}^{k-}\right)=0, \quad k=1, \ldots, s, \\
& \bar{h}(t)=0 \text { a.e. in } \Delta .
\end{aligned}
$$


From the last equation we get

$$
\bar{v}=-H_{v v}^{-1}\left(H_{v x}+f_{v}^{T} Q\right) \bar{x} .
$$

Using this formula in the equation

$$
\dot{\bar{x}}=f_{x} \bar{x}+f_{v} \bar{v}
$$

we see that $\bar{x}$ is a solution of the linear equation

$$
\dot{\bar{x}}=\left(f_{x}-f_{v} H_{v v}^{-1}\left(H_{v x}+f_{v}^{T} Q\right)\right) \bar{x} .
$$

This equation together with initial condition $\bar{x}\left(t_{0}\right)=0$ implies that

$$
\bar{x}(t)=0 \text { for all } t \in\left[t_{0}, t_{1}\right) .
$$

Consequently, $\bar{x}^{1-}=0$, and then $\xi_{1}=0$ by virtue of $(68)$ with $k=1$. The equality $\xi_{1}=0$ together with jump condition $[\bar{x}]^{1}=[\dot{x}]^{1} \xi_{1}$ imply that $[\bar{x}]^{1}=0$, i.e., $\bar{x}$ is continuous at $t_{1}$. Consequently, $\bar{x}^{1+}=0$. From the last condition and equation (71) it follows that

$$
\bar{x}(t)=0 \quad \text { for all } t \in\left(t_{1}, t_{2}\right) .
$$

Repeating this argument we obtain

$$
\xi_{1}=\xi_{2}=\ldots=\xi_{s}=0, \quad \bar{x}(t)=0 \forall t \in\left[t_{0}, t_{f}\right] .
$$

Then from (70) it follows that $\bar{v}=0$. Consequently, we have $\bar{z}=0$ and thus have proved the following theorem.

Theorem 2. Assume that there exists a symmetric matrix $Q(t)$, defined on $\left[t_{0}, t_{f}\right]$, such that

(a) $Q(t)$ is piecewise continuous on $\left[t_{0}, t_{f}\right]$ and continuously differentiable on each interval of the set $\left[t_{0}, t_{f}\right] \backslash \theta$;

(b) $Q(t)$ satisfies the Riccati equation

$$
\dot{Q}+Q f_{x}+f_{x}^{T} Q+H_{x x}-\left(H_{x v}+Q f_{v}\right) H_{v v}^{-1}\left(H_{v x}+f_{v}^{T} Q\right)=0
$$

on each interval of the set $\left[t_{0}, t_{f}\right] \backslash \theta$;

(c) at each point $t_{k} \in \theta$ matrix $Q(t)$ satisfies the jump condition

$$
[Q]^{k}=\left(b_{k-}\right)^{-1}\left(q_{k-}\right)^{T}\left(q_{k-}\right),
$$

where

$$
q_{k-}=\left([\dot{x}]^{k}\right)^{T} Q^{k-}-[\dot{\psi}]^{k}, \quad b_{k-}=D^{k}(H)-\left(q_{k-}\right)[\dot{x}]^{k}>0 ;
$$

(d) $\left\langle\left(l_{x_{f} x_{f}}-Q\left(t_{f}\right)\right) \bar{x}_{f}, \bar{x}_{f}\right\rangle \geq 0$ for all $\bar{x}_{f} \in \mathbb{R}^{d(x)}$ such that

$$
K_{x_{f}}\left(x\left(t_{f}\right)\right) \bar{x}_{f}=0 .
$$

Then $\Omega(\lambda, \bar{z})$ is positive definite on the subspace $\mathscr{K}$. 
Remark. No strict inequality is imposed in the boundary condition (d), since the initial state is fixed. This property easily follows from a perturbation argument; cf., e.g., [11]. When endpoint conditions include inequality constraints of the form $F\left(x\left(t_{f}\right)\right) \leq 0$, then the inequality (d) has to be checked on the cone of elements $\bar{x}_{f} \in \mathbb{R}^{d(x)}$ satisfying $K_{x_{f}}\left(x\left(t_{f}\right)\right) \bar{x}_{f}=0$ and $F_{i, x_{f}}\left(x\left(t_{f}\right)\right) \bar{x}_{f} \leq 0$ if $F_{i}\left(x\left(t_{f}\right)\right)=0$.

In some problems, it is more convenient to integrate the Riccati equation (75) backwards from $t=t_{f}$. A similar proof shows that we can replace condition (c) in Theorem 2 by the following condition:

(c+) at each point $t_{k} \in \theta$ the matrix $Q(t)$ satisfies the jump condition

$$
\begin{aligned}
& {[Q]^{k}=\left(b_{k+}\right)^{-1}\left(q_{k+}\right)^{T}\left(q_{k+}\right),} \\
& \text { where } q_{k+}=\left([\dot{x}]^{k}\right)^{T} Q^{k+}-[\dot{\psi}]^{k}, b_{k+}=D^{k}(H)+\left(q_{k+}\right)[\dot{x}]^{k}>0 .
\end{aligned}
$$

In the next section, we shall discuss an optimal control problem in economics, where all conditions in Theorem 2 can be verified numerically. Let us mention, however, that Theorem 2 is not applicable to the minimum-fuel orbit transfer problem in Oberle, Taubert [16], since the strict Legendre condition (51) does not hold along the zero thrust arc. Nevertheless, Rosendahl [26] has succeeded in deriving second-order sufficient conditions for those controls that belong to a given control structure. For that purpose, the Riccati approach in [11] is extended to multiprocess control problems that are induced by the given control structures.

\section{Numerical Example: Optimal Control of Production and Maintenance}

Cho, Abad and Parlar [4] have introduced an optimal control model where a dynamic maintenance problem is incorporated into a production control problem to simultaneously compute optimal production and maintenance policies. In this model, the dynamics is linear with respect to both production and maintenance control, whereas the cost functional is quadratic with respect to production control and linear with respect to maintenance control. Hence, the model fits into the more general type of control problems considered in (1)-(4). Recently, a detailed numerical analysis for different final times and two types of cost functionals has been given in Maurer, Kim, Vossen [10]. For a certain range of final times, the production control is continuous while the maintenance control is bang-bang. The aim in this section is to show that the sufficient conditions in Theorem 2 are satisfied for the computed solutions.

The notations for state and control variables are different from [4 10] and are chosen in conformity with those in the preceding sections: $x_{1}(t)$ : inventory level at time $t \in$ $\left[0, t_{f}\right]$ with fixed final time $t_{f}>0 ; x_{2}(t)$ : proportion of good units of end items produced at time $t ; v(t)$ : scheduled production rate (control); $m(t)$ : preventive maintenance rate to reduce the proportion of defective units produced (control); $\alpha(t)$ : obsolescence rate of the process performance in the absence of maintenance; $s(t)$ : demand rate; $\rho>0$ : discount rate. The dynamics of the process is given by

$$
\begin{array}{ll}
\dot{x}_{1}(t)=x_{2}(t) v(t)-s(t), & x_{1}(0)=x_{10}>0, \\
\dot{x}_{2}(t)=-\alpha(t) x_{2}(t)+\left(1-x_{2}(t)\right) m(t), x_{2}(0) & =x_{20}>0,
\end{array}
$$


with the following bounds on the control variables,

$$
0 \leq v(t) \leq V, \quad 0 \leq m(t) \leq M \quad \text { for } 0 \leq t \leq t_{f} .
$$

Since all demands must be satisfied, the following state constraint is imposed:

$$
0 \leq x_{1}(t) \text { for } 0 \leq t \leq t_{f}
$$

Computations show that this state constraint is automatically satisfied if we impose the boundary condition

$$
x_{1}\left(t_{f}\right)=0 .
$$

The optimal control problem then is to maximize the total discounted profit plus the salvage value of $x_{2}\left(t_{f}\right)$,

$$
J\left(x_{1}, x_{2}, m, v\right)=\int_{0}^{t_{f}}\left[w s-h x_{1}(t)-r v(t)^{2}-c m(t)\right] e^{-\rho t} d t+b x_{2}\left(t_{f}\right) e^{-\rho t_{f}},
$$

under the constraints $(80)$ - 83 . For later computations, the values of constants are chosen as in Cho et al. [4]:

$$
\begin{aligned}
& s(t) \equiv 4, \alpha(t) \equiv 2, x_{10}=3, x_{20}=1, V=3, M=4, \\
& \rho=0.1, w=8, \quad h=1, \quad c=2.5, b=10, r=2 .
\end{aligned}
$$

In the discussion of the minimum principle (16)-(19), we will not use the current value Hamiltonian function as in [4]10] but will work with the Hamiltonian (Pontryagin) function 11):

$$
\begin{aligned}
H\left(t, x_{1}, x_{2}, \psi_{1}, \psi_{2}, m, v\right) & =e^{-\rho t}\left(-w s+h x_{1}+r v^{2}+c m\right) \\
& +\psi_{1}\left(x_{2} v-s\right)+\psi_{2}\left(-\alpha x_{2}+\left(1-x_{2}\right) m\right),
\end{aligned}
$$

where $\psi_{1}, \psi_{2}$ denote the adjoint variables. The adjoint equations 17 and transversality conditions (18) yield in view of $x_{1}\left(t_{f}\right)=0$ and the salvage term in the cost functional:

$$
\begin{aligned}
& \dot{\psi}_{1}=-h e^{-\rho t}, \quad \psi_{1}\left(t_{f}\right)=\mu, \\
& \dot{\psi}_{2}=-\psi_{1} v+\psi_{2}(\alpha+m), \quad \psi_{2}\left(t_{f}\right)=-b e^{-\rho t_{f}} .
\end{aligned}
$$

The multiplier $\mu$ is not known a priori and will be computed later. We choose a time horizon for which the control constraint $0 \leq v(t) \leq 3$ does not become active. Hence, the minimization in (19) leads to the equation $0=H_{v}=2 r e^{-\rho t} v+\psi_{1} x_{2}$, which yields the control

$$
v=-\psi_{1} x_{2} e^{\rho t} / 2 r
$$

Since the maintenance control enters the Hamiltonian linearly, the control $m$ is determined by the sign of the switching function

$$
\sigma^{m}(t)=H_{m}=e^{-\rho t} c+\psi_{2}(t)\left(1-x_{2}(t)\right)
$$


as the policy

$$
m(t)=\left\{\begin{array}{lll}
M, & \text { if } \quad \sigma^{m}(t)<0 \\
0, & \text { if } \quad \sigma^{m}(t)>0 \\
\text { singular, } & \text { if } \quad \sigma^{m}(t) \equiv 0 \quad \text { for } t \in I_{\text {sing }} \subset\left[0, t_{f}\right]
\end{array}\right\} .
$$

For the final time $t_{f}=1$ which was considered in [4] and [10], the maintenance control contains a singular arc. But the computations in [10] show that for final times $t_{f} \in$ $[0.15,0.98]$ the maintenance control has two bang-bang arcs:

$$
m(t)=\left\{\begin{array}{lll}
0, & \text { for } & 0 \leq t \leq t_{1} \\
M=4, & \text { for } & t_{1}<t \leq t_{f}
\end{array}\right\} .
$$

Let us study the control problem with final time $t_{f}=0.9$ in more detail. To compute a solution candidate, we apply nonlinear programming methods to the discretized control problem with a large number $N$ of grid points $\tau_{i}=i \cdot t_{f} / N, i=0,1, \ldots, N$; cf. [2]3]. We use the modeling language AMPL of Fourer et al. [6], the interior point optimization code IPOPT of Wächter et al. [27] and the integration method of Heun.

For $N=5000$ grid points, the computed state, control and adjoint functions are displayed in Fig. 1 We find the following values for the switching time, functional value and some selected state and adjoint variables:

$$
\begin{array}{rlrl}
t_{1} & =0.65691, & J & =26.705, \\
x_{1}\left(t_{1}\right) & =0.84924, & x_{2}\left(t_{1}\right) & =0.226879, \\
x_{1}\left(t_{f}\right) & =0 ., & x_{2}\left(t_{f}\right) & =0.574104, \\
\psi_{1}(0) & =-7.8617, & \psi_{2}(0) & =-4.70437, \\
\psi_{1}\left(t_{1}\right) & =-8.4975, & \psi_{2}\left(t_{1}\right) & =-3.2016, \\
\psi_{1}\left(t_{f}\right) & =-8.72313, \psi_{2}\left(t_{f}\right) & =-9.13931 .
\end{array}
$$

Let us apply now the second-order sufficient conditions in Theorem 2 Observe first that the sufficiency theorem in Feichtinger and Hartl [5], p. 36, Satz 2.2, is not applicable here. The assumptions in this theorem require that the minimized Hamiltonian $H^{\min }(t, x, \psi(t))$ be convex in the state variable $x=\left(x_{1}, x_{2}\right)$. However, using the minimizing control $v=-\psi_{1} x_{2} e^{\rho t} / 2 r$ from $[88$, we obtain

$$
H^{\min }(t, x, \psi(t))=-\frac{e^{\rho t}}{4 r} \psi_{1}(t)^{2} x_{2}^{2}+L(x),
$$

where $L(x)$ denotes a linear function in the variable $x$. Since $\psi_{1}(t) \neq 0$ for $t \in\left[0, t_{f}\right]$, the minimized Hamiltonian is strictly concave in the variable $x_{2}$. Hence, the sufficiency theorem in [5], Satz 2.2, cannot be used here.

Now we compute the quantities needed in Theorem 2 and (79). The derivative of the switching function $\sigma^{m}(t)=e^{-\rho t} c+\psi_{2}(t)\left(1-x_{2}(t)\right)$ in 89 is given by

$$
\dot{\sigma}^{m}=-\rho e^{-\rho t} c-\psi_{1} v\left(1-x_{2}\right)+\psi_{2} \alpha, \quad v=-\psi_{1} x_{2} e^{\rho t} / 2 r .
$$

Inserting the values given in 92 we get

$$
D^{1}(H)=-4 \dot{\sigma}^{m}\left(t_{1}\right)=27.028>0, \quad \sigma^{m}(t) \neq 0 \quad \forall t \neq t_{1} .
$$


$x_{1}(t)$

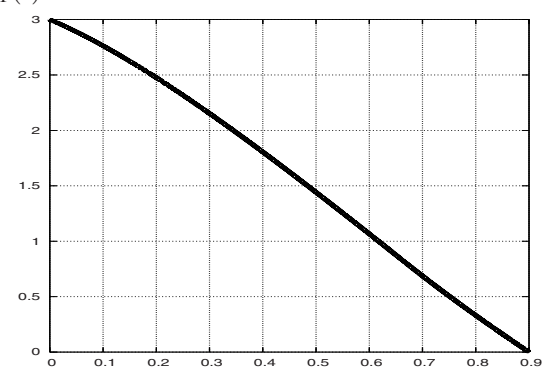

$v(t)$

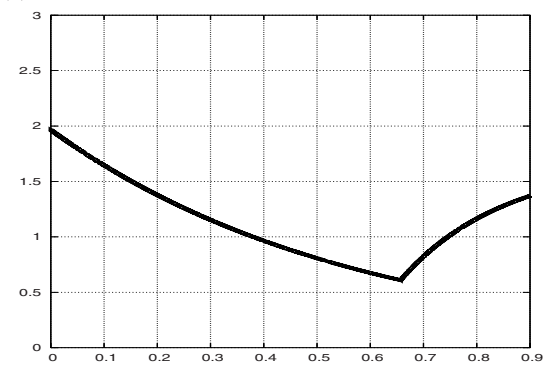

$\psi_{1}(t)$

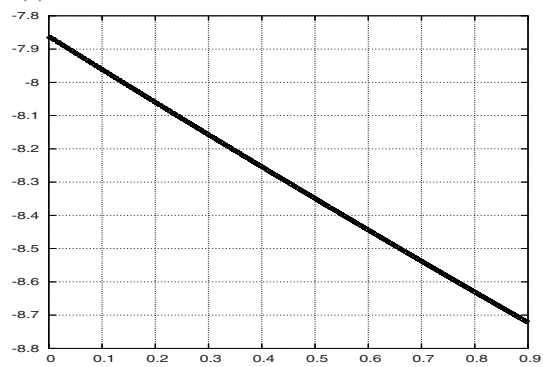

$x_{2}(t)$

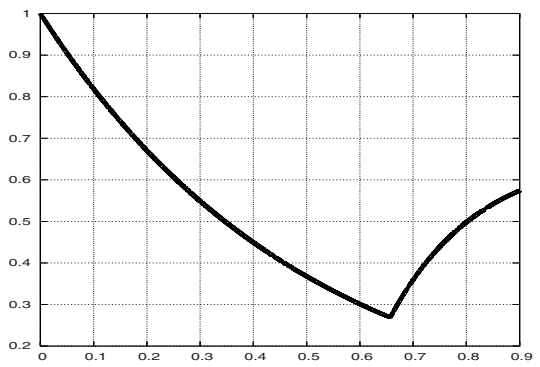

$m(t)$

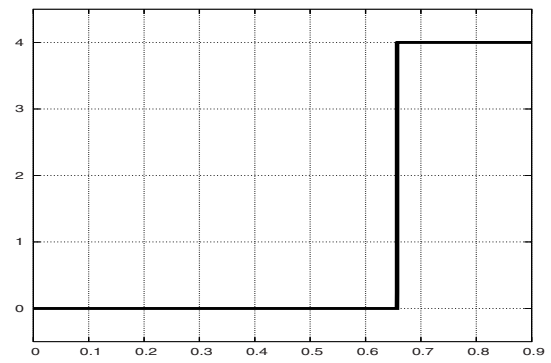

$\psi_{2}(t)$

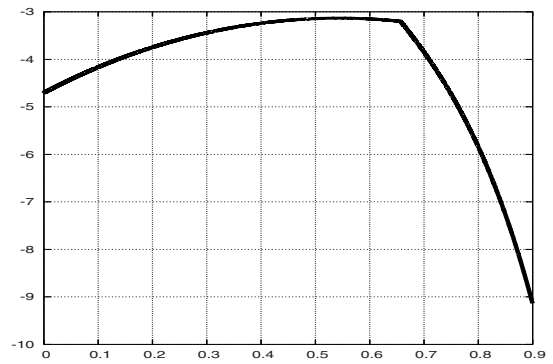

Fig. 1. From above: state variables $x_{1}(t)$ and $x_{2}(t)$; control variables $v(t)$ and $m(t)$; adjoint variables $\psi_{1}(t)$ and $\psi_{2}(t)$

Hence, the maintenance control $m(\cdot)$ is a strict bang-bang control; see Fig 2 Now we evaluate the Riccati equation

$$
\dot{Q}=-Q f_{x}-f_{x}^{T} Q-H_{x x}+\left(H_{x v}+Q f_{v}\right)\left(H_{v v}\right)^{-1}\left(H_{v x}+f_{v}^{T} Q\right)
$$

for the symmetric $2 \times 2$-matrix $Q=\left(\begin{array}{ll}q_{11} & q_{12} \\ q_{12} & q_{22}\end{array}\right)$. Computing the expressions

$$
f_{x}=\left(\begin{array}{cc}
0 & v \\
0 & -(\alpha+m)
\end{array}\right), f_{v}=\left(\begin{array}{c}
x_{2} \\
0
\end{array}\right), H_{x x}=\mathbf{0}, H_{x v}=\left(0, \psi_{1}\right)^{T}, H_{v v}=2 r e^{-\rho t}
$$




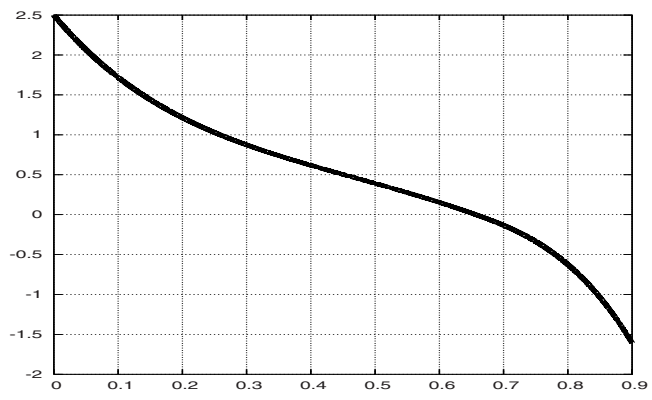

Fig. 2. Switching function $\sigma^{m}(t)$

the matrix Riccati equation (96) yields the following ODE system:

$$
\begin{aligned}
& \dot{q}_{11}=q_{11}^{2} x_{2}^{2} e^{\rho t} / 2 r \\
& \dot{q}_{12}=-q_{11} v+q_{12}(\alpha+m)+e^{\rho t} q_{11} x_{2}\left(\psi_{1}+q_{12} x_{2}\right) / 2 r \\
& \dot{q}_{22}=-2 q_{12} v+2 q_{22}(\alpha+m)+e^{\rho t}\left(\psi_{1}+q_{12} x_{2}\right)^{2} / 2 r .
\end{aligned}
$$

Since equations (98) and 99) are satisfied by zero functions $q_{11}$ and $q_{12}$, we can try to find a solution to the Riccati system with $q_{11}(t)=q_{12}(t) \equiv 0$ on $\left[0, t_{f}\right]$. Then 100 , reduces to the linear equation

$$
\dot{q}_{22}=2 q_{22}(\alpha+m)+e^{\rho t} \psi_{1}^{2} / 2 r .
$$

Obviously, this linear equation has always a solution. The remaining difficulty is to satisfy the jump and boundary conditions in Theorem 2 (c) and (d). Instead of condition (c) we will verify conditions (c+) and (79) which are more convenient for the backward integration of (100). The boundary conditions in Theorem 2 (d) show that the initial value $Q(0)$ can be chosen arbitrarily while the terminal condition imposes the sign condition $q_{22}\left(t_{f}\right) \leq 0$, since $x_{2}\left(t_{f}\right)$ is free. We shall take the boundary condition

$$
q_{22}\left(t_{f}\right)=0
$$

Using the computed values in (92), we solve the linear equation (101) with terminal condition (102). At the switching time $t_{1}$ we obtain the value

$$
q_{22}\left(t_{1}\right)=-1.5599 \text {. }
$$

Next, we evaluate the jump in the state and adjoint variables and will check conditions 79.) We get

$$
\left([\dot{x}]^{1}\right)^{T}=\left(0, M\left(1-x_{2}\left(t_{1}\right)\right)\right), \quad[\dot{\psi}]^{1}=\left(0, M \psi_{2}\left(t_{1}\right)\right),
$$


which yield the quantities

$$
\begin{aligned}
q_{1+} & =\left([\dot{x}]^{1}\right)^{T} Q^{1+}-[\dot{\psi}]^{1}=\left(0, M\left(1-x_{2}\left(t_{1}\right)\right) q_{22}\left(t_{1}+\right)-M \psi_{2}\left(t_{1}\right)\right) \\
& =(0,8.2439), \\
b_{1+} & =D^{1}(H)+\left(q_{1+}\right)[\dot{x}]^{1} \\
& =D^{1}(H)+M^{2}\left(1-x_{2}\left(t_{1}\right)\right)\left(\left(1-x_{2}\left(t_{1}\right)\right) q_{22}\left(t_{1}+\right)-\psi_{2}\left(t_{1}\right)\right) \\
& =27.028+133.55=160.58>0 .
\end{aligned}
$$

Then the jump condition in 179,

$$
[Q]^{1}=\left(b_{1+}\right)^{-1}\left(q_{1+}\right)^{T}\left(q_{1+}\right)=\left(\begin{array}{cc}
0 & 0 \\
0 & {\left[q_{22}\right]^{1}}
\end{array}\right),
$$

reduces to a jump condition for $q_{22}(t)$ at $t_{1}$. However, we do not need to evaluate this jump condition explicitly because the linear equation (101) has a solution regardless of the value $q_{22}\left(t_{1}-\right)$. Hence, we conclude from Theorem 2 that the numerical solution characterized by (92) and displayed in Fig. 1 provides a strict bounded-strong minimum.

Acknowledgements. We are indebted to Kazimierz Malanowski for helpful comments.

\section{References}

1. Agrachev, A.A., Stefani, G., Zezza, P.L.: Strong optimality for a bang-bang trajectory. SIAM J. Control and Optimization 41, 991-1014 (2002)

2. Betts, J.T.: Practical Methods for Optimal Control Using Nonlinear Programming. Advances in Design and Control. SIAM, Philadelphia (2001)

3. Büskens, C., Maurer, H.: SQP-methods for solving optimal control problems with control and state constraints: adjoint variables, sensitivity analysis and real-time control. J. of Computational and Applied Mathematics 120, 85-108 (2000)

4. Cho, D.I., Abad, P.L., Parlar, M.: Optimal production and maintenance decisions when a system experiences age-dependent deterioration. Optimal Control Applic. and Methods 14, 153-167 (1993)

5. Feichtinger, G., Hartl, R.F.: Optimale Kontrolle ökonomischer Prozesse. de Gruyter Verlag, Berlin (1986)

6. Fourer, R., Gay, D.M., Kernighan, B.W.: AMPL: A Modeling Language for Mathematical Programming. Duxbury Press, Brooks-Cole Publishing Company (1993)

7. Hestens, M.: Calculus of Variations and Optimal Control Theory. John Wiley, New York (1966)

8. Malanowski, K.: Stability and sensitivity analysis for optimal control problems with controlstate constraints. Dissertationes Mathematicae, Institute of Mathematics, Polish Academy of Sciences (2001)

9. Maurer, H.: First and second order sufficient optimality conditions in mathematical programming and optimal control. Mathematical Programming Study 14, 163-177 (1981)

10. Maurer, H., Kim, J.-H.R., Vossen, G.: On a state-constrained control problem in optimal production and maintenance. In: Deissenberg, C., Hartl, R.F. (eds.) Optimal Control and Dynamic Games, Applications in Finance, Management Science and Economics, pp. 289308. Springer, Heidelberg (2005) 
11. Maurer, H., Oberle, H.J.: Second order sufficient conditions for optimal control problems with free final time: The Riccati approach. SIAM J. Control and Optimization 41, 380-403 (2002)

12. Maurer, H., Osmolovskii, N.P.: Second order sufficient conditions for time optimal bangbang control problems. SIAM J. Control and Optimization 42, 2239-2263 (2004)

13. Maurer, H., Osmolovskii, N.P.: Second order optimality conditions for bang-bang control problems. Control and Cybernetics 32(3), 555-584 (2003)

14. Maurer, H., Pickenhain, S.: Second-order sufficient conditions for control problems with mixed control-state constraints. Journal of Optimization Theory and Applications 86, 649667 (1995)

15. Milyutin, A.A., Osmolovskii, N.P.: Calculus of Variations and Optimal Control. Translations of Mathematical Monographs, vol. 180. American Mathematical Society, Providence (1998)

16. Oberle, H.J., Taubert, K.: Existence and multiple solutions of the minimum-fuel orbit transfer problem. J. Optimization Theory and Applications 95, 243-262 (1997)

17. Osmolovskii, N.P.: High-order necessary and sufficient conditions for Pontryagin and bounded-strong minima in the optimal control problems. Dokl. Akad. Nauk SSSR, Ser. Cybernetics and Control Theory 303, 1052-1056 (1988); English transl. Sov. Phys. Dokl. 33(12), 883-885 (1988)

18. Osmolovskii, N.P.: Quadratic conditions for nonsingular extremals in optimal control (A theoretical treatment). Russian J. of Mathematical Physics 2, 487-516 (1995)

19. Osmolovskii, N.P.: Second order conditions for broken extremal. In: Ioffe, A., Reich, S., Shafir, I. (eds.) Calculus of Variations and Optimal Control (Technion 1998), pp. 198-216. Chapman and Hall/CRC, Boca Raton (2000)

20. Osmolovskii, N.P.: Second-order sufficient conditions for an extremum in optimal control. Control and Cybernetics 31(3), 803-831 (2002)

21. Osmolovskii, N.P.: Quadratic optimality conditions for broken extremals in the general problem of calculus of variations. Journal of Math. Science 123(3), 3987-4122 (2004)

22. Osmolovskii, N.P., Lempio, F.: Jacobi-type conditions and Riccati equation for broken extremal. Journal of Math. Science 100(5), 2572-2592 (2000)

23. Osmolovskii, N.P., Lempio, F.: Transformation of quadratic forms to perfect squares for broken extremals. Journal of Set Valued Analysis 10, 209-232 (2002)

24. Osmolovskii, N.P., Maurer, H.: Equivalence of second order optimality conditions for bangbang control problems. Part 1: Main results. Control and Cybernetics 34, 927-950 (2005); Part 2: Proofs, variational derivatives and representations. Control and Cybernetics 36, 5-45 (2007)

25. Pontryagin, L.S., Boltyanskii, V.G., Gamkrelidze, R.V., Mishchenko, E.F.: The Mathematical Theory of Optimal Processes. Fizmatgiz, Moscow; English translation: Pergamon Press, New York (1964)

26. Rosendahl, R.: Second order sufficient conditions for space-travel optimal control problems. Talk presented at the 23rd IFIP TC7 Conference on Systems Modelling and Optimization, Cracow, Poland (2007)

27. Wächter, A., et al.: http://projects.coin-or.org/Ipopt

28. Zeidan, V.: The Riccati equation for optimal control problems with mixed state control problems: necessity and sufficiency. SIAM Journal on Control and Optimization 32, 1297-1321 (1994) 